\title{
SUBGROUPS OF PROFINITE SURFACE GROUPS
}

\author{
Lior Bary-Soroker, Katherine F. Stevenson, and Pavel A. Zalesskit
}

\begin{abstract}
We study the subgroup structure of the étale fundamental group $\Pi$ of a projective curve over an algebraically closed field of characteristic 0 . We obtain an analog of the diamond theorem for $\Pi$. As a consequence we show that most normal subgroups of infinite index are semi-free. In particular every proper open subgroup of a normal subgroup of infinite index is semi-free.
\end{abstract}

\section{Introduction}

Every subgroup of a free group is free, this is the content of the Nielsen-Schreier theorem. The profinite version of the Nielsen-Schreier theorem fails in general and even fails for normal subgroups, for example $\mathbb{Z}_{p} \leq \widehat{\mathbb{Z}}$. Therefore the question of finding conditions under which a subgroup of a free profinite group is free is natural and of importance. The question was considered by Melnikov, Lubotzky, van der Dries, Jarden, Haran, and others ([7, Chapter 8] and [3, Chapter 25]).

Roughly speaking the most general criteria are Melnikov's characterization of normal (and accessible) subgroups of free profinite groups and Haran's diamond theorem. In this work we consider the étale fundamental group $\Pi=\pi_{1}(X)$, where $X$ is a curve over an algebraically closed field of characteristic 0 and the genus of its smooth completion is $\geq 2$.

If $X$ is affine, then $\Pi$ is free of finite rank. Therefore Melnikov's characterization is known to hold [7, Chapter 8.6] and similarly Haran's diamond theorem [1. If $X$ is projective, then $\Pi$ is a profinite surface group, i.e., the profinite completion of a surface group. Melnikov's characterization for normal subgroups of $\Pi$ is obtained in [8. The objective of this work is to obtain the diamond theorem for profinite surface groups:

Theorem 1.1. Let $\Pi$ be a profinite surface group of genus $g \geq 2$ and let $N$ be a closed subgroup of $\Pi$ with $[\Pi: N]=\prod_{p} p^{\infty}$ as supernatural numbers, where $p$ runs over all primes. Assume there exist closed normal subgroups $K_{1}, K_{2}$ of $\Pi$ such that $K_{1} \cap K_{2} \leq N$ but $K_{1} \not \leq N$ and $K_{2} \not \leq N$. Then $N$ is a free profinite group of countable rank.

We note that a necessary condition for a profinite group to be free is that it is projective, and a closed subgroup $N$ of a profinite surface group $\Pi$ is projective if and only if $[\Pi: N]=\prod_{p} p^{\infty}$ as supernatural numbers, where $p$ runs over all primes $[8$, Proposition 1.2].

Recently a notion of "free not necessarily projective" profinite groups evolved from Galois theory [5, 2], the so called semi-free groups. A second countable profinite group

Received by the editors November 5, 2010.

1991 Mathematics Subject Classification. 20E18, 20F34, 57M05. 
is semi-free if and only if every finite split embedding problem is properly solvable. Using this notion we can generalize Theorem 1.1 to any closed subgroup of infinite index:

Theorem 1.2. Let $\Pi$ be a profinite surface group of genus $g \geq 2$ and let $N$ be a closed subgroup with $[\Pi: N]=\infty$. Assume there exist closed normal subgroups $K_{1}, K_{2}$ of $\Pi$ such that $K_{1} \cap K_{2} \leq N$ but $K_{1} \not \leq N$ and $K_{2} \not \leq N$. Then $N$ is semi-free of countable rank.

Since a semi-free projective group is free [2, Theorem 3.6], Theorem 1.1 follows from Theorem 1.2 .

A consequence of Theorem 1.2 is that 'most' closed normal subgroups of $\Pi$ of infinite index are semi-free in the following sense.

Corollary 1.3. Let $\Pi$ be a profinite surface group of genus $g \geq 2$ and let $N$ be a closed normal subgroup with $[\Pi: N]=\infty$. Then every proper open subgroup of $N$ is semi-free.

We give more examples in Section 4.3 .

A typical example of a closed normal subgroup which is not semi-free is the kernel $M$ of the epimorphism from $\Pi$ to its maximal pro- $p$ quotient (because $M$ has no $p$ quotient). Note however that $M$ is contained in a semi-free closed normal subgroup of $\Pi$. Indeed, there exists a continuous epimorphism $\alpha: \Pi \rightarrow \mathbb{Z}_{p}^{2}$, so $\operatorname{ker} \alpha=K_{1} \cap K_{2}$, where $K_{1}, K_{2}$ are closed normal subgroups of $\Pi$ with $\Pi / K_{i} \cong \mathbb{Z}_{p}$. By Theorem 1.2 $\operatorname{ker} \alpha$ is semi-free, and clearly $M \leq \operatorname{ker} \alpha$.

We show in fact that every closed normal subgroup $N$ of $\Pi$ of infinite index such that $\Pi / N$ is not hereditarily just infinite is contained in a semi-free closed normal subgroup. (An infinite profinite group is just infinite if it has no proper infinite quotient. It is hereditarily just infinite if every open normal subgroup of it is just infinite.)

Theorem 1.4. Let $\Pi$ be a profinite surface group of genus $g \geq 2$ and let $N$ be a closed normal subgroup with $[\Pi: N]=\infty$ such that $\Pi / N$ is not hereditarily just infinite. Then there exists a semi-free closed normal subgroup $M$ of $\Pi$ such that $N \leq M$.

Through the paper, all subgroups of a profinite group are assumed to be closed, and all homomorphisms of profinite groups are assumed to be continuous. The paper is organized as follows. Section 2 is dedicated to preliminary results on surface groups. In Section 3 we prove Theorems 1.1 and 1.2 . In the last section we prove Corollary 1.3 and Theorem 1.4

\section{Surface groups}

The fundamental group $\pi_{1}(X)$ of an oriented Riemann surface $X$ of genus $g$ is given by the presentation

$$
\pi_{1}(X)=\left\langle x_{1}, \ldots, x_{g}, y_{1}, \ldots, y_{g} \mid \prod_{i=1}^{g}\left[x_{i}, y_{i}\right]\right\rangle .
$$

Here $[x, y]=x^{-1} y^{-1} x y$. A group with this presentation is said to be a surface group of genus $g$. We shall call its profinite completion $\Pi$ a profinite surface group of genus $g$. Note that $\Pi$ is the same as the ètale fundamental group of $X$. 
Fact 2.1. Let $\Pi$ be a profinite surface group of genus $g$ and let $U$ be an open subgroup of index $n$. Then $U$ is a profinite surface group of genus $n(g-1)+1$.

Proof. This is well known for surface groups. The map $\pi_{1}(X) \rightarrow \Pi$ induces an indexpreserving bijection between finite index subgroups of $\pi_{1}(X)$ and open subgroups of $\Pi$ sending a subgroup to its completion [7, Chapter 3].

Let $\Pi$ be a profinite group. A finite split embedding problem (FSEP) for $\Pi$ consists of finite groups $A, B$ and epimorphisms $f: \Pi \rightarrow B$ and $\alpha: A \rightarrow B$, such that $\alpha$ splits. We denote it by $(f, \alpha)$. A weak solution is a homomorphism $\psi: \Pi \rightarrow A$ such that $\alpha \circ \psi=f$. If $\psi$ is surjective we say it is a proper solution.

We shall need the following technical lemma.

Lemma 2.2. Let $(f: \Pi \rightarrow B, \alpha: A \rightarrow B)$ be a finite split embedding problem for $\Pi$ of genus $g \geq 2|A|^{3}$. Then $(f, \alpha)$ is properly solvable.

Remark 2.3. The bound $g \geq 2|A|^{3}$ is not the best possible. In fact, if $s$ is the minimal number of generators of ker $\alpha$ as a normal subgroup of $A$, then $g \geq s|B|^{2}(|A|+1)$ suffices. We will not use this sharper bound here, and hence will not prove it.

Proof. Let $n=|A|$, and $\beta: B \rightarrow A$ a section of $\alpha$. Note that ker $\alpha$ is generated by $\frac{|A|}{|B|}$ elements. Let $\varphi=\beta \circ f: \Pi \rightarrow A$. Then $\varphi$ is a weak solution.

By [6. Lemma 6.1], it suffices to replace the generators of $\Pi$ with a different set of generators having the same unique relation such that the first $\frac{|A|^{2}+|A|}{|B|} \leq \frac{2|A|^{2}}{|B|}$ new $x_{i}$ 's (resp., $y_{i}$ 's) have the same image under $\varphi$. Let $r=\frac{2|A|^{2}}{|B|}$.

Each of the $g$ pairs $\left(x_{i}, y_{i}\right)$ has $|B|^{2}$ possibilities for $\left(\varphi\left(x_{i}\right), \varphi\left(y_{i}\right)\right)$, hence, since $g \geq 2|A|^{3} \geq|B|^{2} r$, Dirichlet's box principle gives indexes $j_{1}<\cdots<j_{r}$ for which

$$
\varphi\left(x_{j_{1}}\right)=\cdots=\varphi\left(x_{j_{r}}\right) \quad \text { and } \quad \varphi\left(y_{j_{1}}\right)=\cdots=\varphi\left(y_{j_{r}}\right)
$$

The following argument explains how to replace $j_{1}$ with $1, j_{2}$ with 2 , and so forth. Let $x^{y}=y^{-1} x y$. Suppose $j_{1} \neq 1$. Then

$$
\prod_{i=1}^{g}\left[x_{i}, y_{i}\right]=\left[x_{j_{1}}, y_{j_{1}}\right]\left(\left[x_{1}, y_{1}\right] \cdots\left[x_{j_{1}-1}, y_{j_{1}-1}\right]\right)^{\left[x_{j_{1}}, y_{j_{1}}\right]}\left[x_{j_{1}+1}, y_{j_{1}+1}\right] \cdots\left[x_{g}, y_{g}\right] \text {. }
$$

For each $i=1, \ldots, j_{1}-1$, replace the pair of generators $x_{i}, y_{i}$ with $x_{i}^{\left[x_{j_{1}}, y_{j_{1}}\right]}, y_{i}^{\left[x_{j_{1}}, y_{j_{1}}\right]}$. Thus we may assume that $j_{1}=1$. Continuing similarly, we get a new presentation of $\Pi$ of the same kind for which (1) holds, and hence by [6, Lemma 6.1] $(f, \alpha)$ is solvable.

\section{Diamond $\diamond$}

In this section we prove Theorems 1.2 and 1.1 .

3.1. Haran-Shapiro Induction. Let $N \leq \Pi$ be a subgroup of $\Pi$. Consider a FSEP

$$
\mathcal{E}=\left(\mu_{1}: N \rightarrow G_{1}, \alpha_{1}: A \rtimes G_{1} \rightarrow G_{1}\right)
$$

for $N$. We describe a method to construct an embedding problem $\mathcal{E}_{\text {ind }}$ for $\Pi$ such that a weak solution of $\mathcal{E}_{\text {ind }}$ induces a weak solution of $\mathcal{E}$, and under certain conditions, a proper solution of $\mathcal{E}_{\text {ind }}$ induces a proper solution of $\mathcal{E}$. 
We start by setting up the notation. Let $L \triangleleft \Pi$ be an open normal subgroup of $\Pi$. Assume

$$
L \cap N \leq \operatorname{ker} \mu_{1}
$$

Let $\mu: \Pi \rightarrow G:=\Pi / L$ be the natural epimorphism, $G_{0}=N L / L \cong N / N \cap L$, and $\mu_{0}=\left.\mu\right|_{N}: N \rightarrow G_{0}$. Then $\mu_{1}$ factors as $\mu_{1}=\nu \circ \mu_{0}$, where $\nu: G_{0} \rightarrow G_{1}$ is induced by (2). The group $G_{0}$ acts on $A$ via $\nu$, i.e., $a^{g}:=a^{\nu(g)}$, for all $a \in A, g \in G_{0}$.

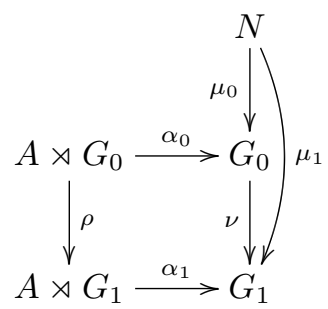

Here $\rho(a, g)=(a, \nu(g))$ and $\alpha_{0}$ is the projection map. The group $G$ acts on

$$
\operatorname{Ind}_{G_{0}}^{G}(A)=\left\{f: G \rightarrow A \mid f(\sigma \tau)=f(\sigma)^{\tau}, \forall \sigma \in G, \tau \in G_{0}\right\} \cong A^{\left(G: G_{0}\right)}
$$

by $\left(f^{\sigma}\right)\left(\sigma^{\prime}\right)=f\left(\sigma \sigma^{\prime}\right)$, for all $\sigma, \sigma^{\prime} \in G, f \in \operatorname{Ind}_{G_{0}}^{G}(A)$. This gives rise to the so called twisted wreath product

$$
A \imath_{G_{0}} G=\operatorname{Ind}_{G_{0}}^{G}(A) \rtimes G .
$$

Let $\alpha: A \imath_{G_{0}} G \rightarrow G$ be the projection map. Then we have the following FSEP for $\Pi$ induced from $\mathcal{E}$ (w.r.t. $L$ satisfying $(2)$ ):

$$
\mathcal{E}_{\text {ind }}(L)=\left(\mu: \Pi \rightarrow G, \alpha: A \imath_{G_{0}} G \rightarrow G\right) .
$$

Let Sh: $\operatorname{Ind}_{G_{0}}^{G}(A) \rtimes G_{0} \rightarrow A \rtimes G_{0}$ be defined by $\operatorname{Sh}((f, \sigma))=f(1) \sigma$. Clearly Sh is surjective, it is also a homomorphism, since

$$
\operatorname{Sh}\left(f^{\sigma}\right)=f^{\sigma}(1)=f(\sigma)=f(1)^{\sigma}=\operatorname{Sh}(f)^{\sigma} .
$$

Now, a weak solution $\psi: \Pi \rightarrow A \imath_{G_{0}} G$ of $\mathcal{E}_{\text {ind }}$ induces the weak solution $\psi^{\text {ind }}=$ $\left.\rho \circ \operatorname{Sh} \circ \psi\right|_{N}$ of $\mathcal{E}$ :

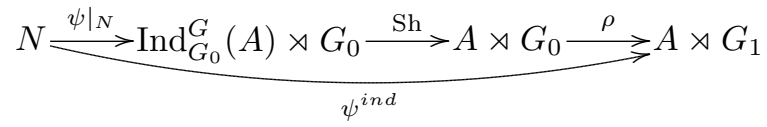

(Note $\psi(N) \leq \operatorname{Ind}_{G_{0}}^{G}(A) \rtimes G_{0}$ since $\mu(N)=\mu_{0}(N)=G_{0}$, hence Sh $\left.\circ \psi\right|_{N}$ is well defined.)

Assume $\psi$ is surjective. In general this does not imply surjectivity of $\psi^{\text {ind }}$. The following result gives a working sufficient condition on $L$ for $\psi^{\text {ind }}$ to be surjective.

Proposition 3.1 ([2, Proposition 4.5]). Let $N \leq \Pi$ be profinite groups and let

$$
\mathcal{E}=\left(\mu_{1}: N \rightarrow G_{1}, \alpha_{1}: A \rtimes G_{1} \rightarrow G_{1}\right)
$$

be a FSEP for $N$. Let $D, \Pi_{0}, L$ be subgroups of $\Pi$ such that

(4a) $D$ is an open normal subgroup of $\Pi$ with $N \cap D \leq \operatorname{ker} \mu_{1}$,

(4b) $\Pi_{0}$ is an open subgroup of $\Pi$ with $N \leq \Pi_{0} \leq N D$,

(4c) $L$ is an open normal subgroup of $\Pi$ with $L \leq \Pi_{0} \cap D$. 
In particular $L \cap N \leq D \cap N \leq \operatorname{ker} \mu_{1}$, so (2) holds. Let $\mathcal{E}_{\text {ind }}$ be as in (3).

Assume that there is a closed normal subgroup $\mathcal{N}$ of $\Pi$ with $\mathcal{N} \leq N \cap L$ such that there is $N O$ nontrivial quotient $\bar{A}$ of $A$ through which the action of $G_{0}$ on $A$ descends and for which the FSEP

$$
\overline{\mathcal{E}}_{i n d, \mathcal{N}}(L)=\left(\bar{\mu}: \Pi / \mathcal{N} \rightarrow G, \bar{\alpha}: \bar{A} \imath_{G_{0}} G \rightarrow G\right),
$$

where $\bar{\mu}$ is the quotient map, $G=\Pi / L$, and $G_{0}=\Pi_{0} / L$, is properly solvable. Then a proper solution $\psi$ of $\mathcal{E}_{\text {ind }}$ induces a proper solution $\psi^{\text {ind }}$ of $\mathcal{E}$.

3.2. Condition $(\diamond)$. Let $N \leq \Pi$ be profinite groups with $[\Pi: N]=\infty$ and assume there exist normal subgroups $N_{1}, N_{2}$ of $\Pi$ such that $N_{1} \cap N_{2} \leq N$ but $N_{1} \not \leq N$ and $N_{2} \not \leq N$. Without loss of generality we can assume that $\left[N_{1} N: N\right]=\infty$. Indeed, if $\left[N_{1} N: N\right]<\infty$, then $\Pi$ has an open subgroup $N_{2}^{\prime}$ such that $N_{2}^{\prime} \cap\left(N_{1} N\right) \leq N$. Then $N_{1} \cap N_{2}^{\prime} \leq N$ and $\left[N_{2}^{\prime} N: N\right]=\infty$. Replace $N_{1}$ with $N_{2}^{\prime}$ and $N_{2}$ with $N_{1}$ to get the assumption.

The following result will be used in the sequel.

Lemma 3.2. Let $N \leq \Pi$ be profinite groups with $[\Pi: N]=\infty$ and assume there exist normal subgroups $N_{1}, N_{2}$ of $\Pi$ such that $N_{1} \cap N_{2} \leq N,\left[N_{1}: N_{1} \cap N\right]=\infty$, and $N_{2} \not \leq N$. Let

$$
\mathcal{E}=\left(\mu_{1}: N \rightarrow G_{1}, \alpha_{1}: A \rtimes G_{1} \rightarrow G_{1}\right)
$$

be a FSEP for $N$. Let $L$ be an open normal subgroup of $\Pi$ satisfying

(i) $L \cap N \leq \operatorname{ker} \mu_{1}$,

(ii) $\left[N_{1} N L: N L\right] \geq 3$,

(iii) $\left[N_{2} N L: N L\right] \geq 2$, and

(iv) $[\Pi: N L] \geq 3$.

Let $G=\Pi / L, G_{0}=N L / L \cong N / N \cap L$ and let

$$
\mathcal{E}_{\text {ind }}=\left(\mu: \Pi \rightarrow G, \alpha: A \text { ใ }_{G_{0}} G \rightarrow G\right)
$$

be as defined the induced embedding problem of Equation (3). Then a proper solution $\psi$ of $\mathcal{E}_{\text {ind }}$ induces a proper solution $\psi^{\text {ind }}$ of $\mathcal{E}$.

Proof. To prove the assertion we use Proposition 3.1. Choose an open normal subgroup $D$ of $\Pi$ with $N \cap D \leq \operatorname{ker} \mu_{1}$, let $\Pi_{0}=N D$. We can choose an open normal subgroup $L$ of $\Pi$ such that

$$
\begin{aligned}
& N_{1} L, N_{2} L \not \leq N L\left(\text { use } N_{1}, N_{2} \not \leq N\right), \\
& {[\Pi: N L]>2(\text { use }[\Pi: N]>2),} \\
& \left(N_{1} N L: N L\right)>2\left(\text { use }\left[N_{1} N: N\right]>2\right) .
\end{aligned}
$$

Let $G=\Pi / L, \mu: \Pi \rightarrow G$ be the natural epimorphism, $G_{0}=\mu(N)=N L / L \cong$ $N / N \cap L$, and $G_{i}=\mu\left(N_{i}\right)=N_{i} L / L \cong N_{i} / N_{i} \cap L$. Then taking the above conditions modulo $L$ gives the following conditions.

$$
\begin{aligned}
& G_{1}, G_{2} \not \leq G_{0} . \\
& {\left[G: G_{0}\right]>2 .} \\
& {\left[G_{1} G_{0}: G_{0}\right]>2 .}
\end{aligned}
$$

Let $\mathcal{N}=N_{1} \cap N_{2} \cap L$. 
Let $\bar{A}$ be a non-trivial quotient of $A$ through which the action of $G_{0}$ descends. By Proposition 3.1 it suffices to show that $\overline{\mathcal{E}}_{i n d, \mathcal{N}}$ appearing in $(5)$ is not properly solvable.

Assume $\psi: \Pi \rightarrow \bar{A} \imath_{G_{0}} G$ is an epimorphism with $\alpha \circ \psi=\mu$ that factors through $\Pi / \mathcal{N}$. Then $\psi(\mathcal{N})=1$. For $i=1,2$ put $H_{i}=\psi\left(N_{i}\right)$. Then $H_{i} \triangleleft \bar{A} \imath_{G_{0}} G$ and $\alpha\left(H_{i}\right)=\mu\left(N_{i}\right)=G_{i}$. By (6) there is an $h_{2} \in H_{2}$ with $\alpha\left(h_{2}\right) \notin G_{0}$. Recalling (8), [3, Lemma 13.7.4(a)] gives an $h_{1} \in H_{1}$ for which $\alpha\left(h_{1}\right)=1$ and $\left[h_{1}, h_{2}\right] \neq 1$.

For $i=1,2$, lift $h_{i}$ to $y_{i} \in N_{i}$ (i.e., $\left.\psi\left(y_{i}\right)=h_{i}\right)$. Then $\mu\left(y_{1}\right)=\alpha\left(h_{1}\right)=1$. So, $y_{1} \in L$. Then $\left[y_{1}, y_{2}\right] \in\left[L, N_{2}\right] \cap\left[N_{1}, N_{2}\right] \leq L \cap\left(N_{1} \cap N_{2}\right)=\mathcal{N}$. So, $\left[h_{1}, h_{2}\right]=$ $\left[\psi\left(y_{1}\right), \psi\left(y_{2}\right)\right] \in \psi(\mathcal{N})=1$. This contradiction proves that $\psi$ as above does not exist.

We write $f \uparrow \infty$ for an increasing function $f: \mathbb{R}^{+} \rightarrow \mathbb{R}^{+}$with $\lim _{x \rightarrow \infty} f(x)=\infty$.

We say that a subgroup $N$ of $\Pi$ with $[\Pi: N]=\infty$ satisfies Condition $(\diamond)$ in $\Pi$ if there exist normal subgroups $N_{1}, N_{2}$ of $\Pi$ such that $N_{1} \cap N_{2} \leq N,\left[N_{1}: N_{1} \cap N\right] \geq 3$, $\left[N_{2}: N_{2} \cap N\right] \geq 2$, and for every $f \uparrow \infty, r \in \mathbb{N}$, and open subgroup $N^{\prime}$ of $N$ there exists a diagram of subgroups

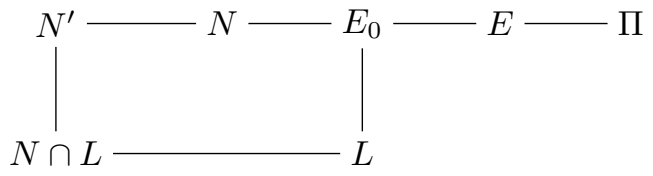

such that

(9a) $L \leq E_{0} \leq E$ are open in $\Pi$;

(9b) $L$ is normal in $E$;

(9c) $\left[N_{1} \cap E: N_{1} \cap E_{0}\right] \geq 3$;

(9d) $\left[N_{2} \cap E: N_{2} \cap E_{0}\right] \geq 2$;

(9e) $f([\Pi: E]) \geq r \cdot[E: L]$.

In the sequel we use the notion of sparse and abundant subgroups ([1, Defintion 2.1]) and some of their basic properties.

Definition 3.3. A closed subgroup $M$ of a profinite group $\Pi$ of infinite index is called sparse if for every $n \in \mathbb{N}$ there exists an open subgroup $K$ of $\Pi$ containing $M$ such that for every proper open subgroup $L$ of $K$ containing $M$ we have $[K: L] \geq n$.

It follows that one can take $K$ with arbitrarily large index in $\Pi$. See [2, Definition 5.1].

A subgroup of $\Pi$ is called abundant if it is not open and not sparse

Proposition 3.4. Let $\Pi, N, N_{1}, N_{2}$ be profinite groups such that $N, N_{1}, N_{2}$ are sub-

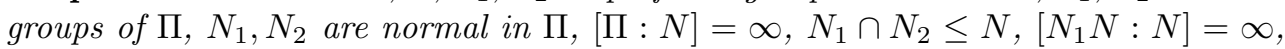
and $N_{2} \not \leq N$. Each of the following implies that $N$ satisfies Condition $(\diamond)$ in an open subgroup of $\Pi$.

(10a) $\left[\Pi: N N_{1} N_{2}\right]=\infty$.

(10b) $\left[\Pi: N N_{1} N_{2}\right]<\infty$ and $N N_{1}$ is abundant in $\Pi$.

(10c) $\left[\Pi: N N_{1} N_{2}\right]<\infty$ and $N N_{2}$ is abundant in $\Pi$.

(10d) $\left[\Pi:\left(N N_{1}\right) \cap\left(N N_{2}\right)\right]<\infty$ and $N$ is abundant in $\Pi$. 
We need two lemmas for the proof.

Lemma 3.5. Let $\Pi$ be a profinite group and $N$ a subgroup of $\Pi$ of infinite index. Let $N_{1}, N_{2}$ be normal subgroups of $\Pi$ such that $N_{1} \cap N_{2} \leq N,\left[N_{1}: N_{1} \cap N\right] \geq 3$ and $\left[N_{2}: N_{2} \cap N\right] \geq 2$. Assume that for every $f \uparrow \infty, s \in \mathbb{N}$, $\Pi$ has open subgroups $E_{1} \leq E$ containing $N$ such that $f([\Pi: E]) \geq s \cdot\left[E: E_{1}\right]$ ! and for each $i \in\{1,2\}$ either

(11a) $N_{i} \leq E$ or

(11b) $N_{i} E_{1}=\Pi$ and $\left[E: E_{1}\right] \geq 3$.

Then $N$ satisfies Condition $(\diamond)$.

Proof. Let $f \uparrow \infty, r \in \mathbb{N}$ and $N^{\prime}$ an open subgroup of $N$. Then there exists an open normal subgroup $D$ of $\Pi$ such that $D \cap N \leq N^{\prime}$. Since $\left[N_{1}: N_{1} \cap N\right] \geq 3$, and $\left[N_{2}: N_{2} \cap N\right] \geq 2$, $\Pi$ has an open normal subgroup $H$ containing $N$ such that

$$
\left[N_{1}: N_{1} \cap H\right] \geq 3 \quad \text { and } \quad\left[N_{2}: N_{2} \cap H\right] \geq 2 .
$$

Put $s=r \cdot[\Pi: H] ![\Pi: D]$.

Our condition gives open subgroups $E_{1} \leq E$ containing $N$ such that $f([\Pi: E]) \geq$ $s \cdot\left[E: E_{1}\right]$ ! and for each $i \in\{1,2\}$ either $11 \mathrm{a}$ or $11 \mathrm{~b}$ holds. Set $E_{0}=H \cap E_{1}$. Let $E_{11}=\bigcap_{\sigma \in E} E_{1}^{\sigma}$ (resp., $H_{00}=\bigcap_{\sigma \in \Pi} H^{\sigma}$ ) be the normal core of $E_{1}$ (resp., $H$ ) in $E$ (resp., П). Finally let $L=H_{00} \cap E_{11} \cap D$. Then $L \leq H_{00} \cap E_{11} \leq H \cap E_{1}=E_{0}$.

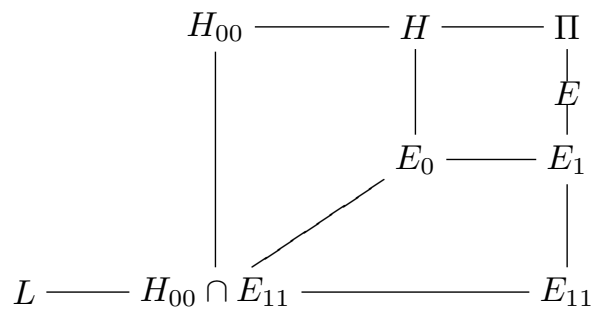

We have

$$
\begin{aligned}
{[E: L] } & =\left[E: H_{00} \cap E_{11} \cap D\right] \\
& =\left[E: E_{11}\right]\left[E_{11}: H_{00} \cap E_{11}\right]\left[H_{00} \cap E_{11}: H_{00} \cap E_{11} \cap D\right] \\
& \leq\left[E: E_{11}\right]\left[\Pi: H_{00}\right][\Pi: D] \\
& \leq\left[E: E_{1}\right] ![\Pi: H] ![\Pi: D] \leq \frac{1}{s} f([\Pi: E])[\Pi: H] ![\Pi: D] \\
& =\frac{1}{r} f([\Pi: E]) .
\end{aligned}
$$

It remains to show that $\left[N_{1} \cap E: N_{1} \cap E_{0}\right] \geq 3$ and $\left[N_{2} \cap E: N_{2} \cap E_{0}\right] \geq 2$. First assume (11a), i.e., $N_{i} \leq E$. Then, since $E_{0} \leq H$,

$$
\left[N_{i} \cap E: N_{i} \cap E_{0}\right] \geq\left[N_{i}: N_{i} \cap H\right],
$$

and we are done by 12 .

Next assume (11b), i.e., $N_{i} E_{1}=\Pi$ and $\left[E: E_{1}\right] \geq 3$. Then $\left(N_{i} \cap E\right) E_{1}=E$, so

$$
\left[N_{i} \cap E: N_{i} \cap E_{0}\right] \geq\left[N_{i} \cap E: N_{i} \cap E_{1}\right]=\left[E: E_{1}\right]
$$

as needed. 
Lemma 3.6. Let $N$ be an abundant subgroup of a profinite group $\Pi$. Then for every $f \uparrow \infty$ and $s \in \mathbb{N}$ there exist open subgroups $N \leq E_{1} \leq E \leq \Pi$ such that $f([\Pi: E]) \geq s \cdot\left[E: E_{1}\right] !$ and $\left[E: E_{1}\right] \geq 3$.

Proof. Since $N$ is abundant in $\Pi$, there exist $m, n \in \mathbb{N}$ such that for every open subgroup $\Pi_{0}$ of $\Pi$ containing $N$ with $\left[\Pi: \Pi_{0}\right] \geq m$ there exists an open subgroup $\Pi_{1}$ of $\Pi_{0}$ containing $N$ such that $1<\left[\Pi_{0}: \Pi_{1}\right] \leq n$.

Let $f \uparrow \infty$ and $s \in \mathbb{N}$. By definition, $[\Pi: N]=\infty$. Thus there exists an open subgroup $\Pi_{0}$ of $\Pi$ containing $N$ with $f\left(\left[\Pi: \Pi_{0}\right]\right) \geq \max \{s \cdot n !, s \cdot 4 !, f(m)\}$. In particular $f\left(\left[\Pi: \Pi_{0}\right]\right) \geq f(m)$, thus $\left[\Pi: \Pi_{0}\right] \geq m$. By assumption, $\Pi_{0}$ has an open subgroup $\Pi_{1}$ containing $N$ such that $1<\left[\Pi_{0}: \Pi_{1}\right] \leq n$.

If $\left[\Pi_{0}: \Pi_{1}\right] \geq 3$, then the subgroups $E=\Pi_{0}$ and $E_{1}=\Pi_{1}$ satisfy the conclusion of the lemma because $f\left(\left[\Pi: \Pi_{0}\right]\right) \geq s \cdot n$ !. Otherwise, $\left[\Pi_{0}: \Pi_{1}\right]=2$. By assumption $\Pi_{1}$ has an open subgroup $\Pi_{2}$ containing $N$ such that $1<\left[\Pi_{1}: \Pi_{2}\right] \leq n$. If $\left[\Pi_{1}: \Pi_{2}\right] \geq 3$, then, similarly to the previous case, the subgroups $E=\Pi_{1}$ and $E_{1}=\Pi_{2}$ satisfy the conclusion of the lemma. Otherwise, $\left[\Pi_{1}: \Pi_{2}\right]=2$, thus $\left[\Pi_{0}: \Pi_{2}\right]=4$, so $E=\Pi_{0}$, $E_{1}=\Pi_{2}$ satisfy the conclusion of the lemma because $f\left(\left[\Pi: \Pi_{0}\right]\right) \geq s \cdot 4$ !. .

Proof of Proposition 3.4. Let $f \uparrow \infty$ and $s \in \mathbb{N}$. By Lemma 3.5 it suffices to find open subgroups $E_{1} \leq E$ of $\Pi$ containing $N$ such that $f([\Pi: E]) \geq s \cdot\left[E: E_{1}\right]$ ! and for each $i \in\{1,2\}$ either

(i) $N_{i} \leq E$ or

(ii) $N_{i} E_{1}=\Pi$ and $\left[E: E_{1}\right] \geq 3$.

We distinguish between the four cases:

In the first case we have $\left[\Pi: N N_{1} N_{2}\right]=\infty$. Then there exists an open subgroup $E$ of $\Pi$ containing $N N_{1} N_{2}$ such that $f([\Pi: E]) \geq s$. Put $E_{1}=E$. Then $N_{1}, N_{2} \leq E$ and $f([\Pi: E]) \geq s \cdot\left[E: E_{1}\right]$ !.

In the second case, we assume that $\left[\Pi: N N_{1} N_{2}\right]<\infty$ and $N N_{1}$ is abundant in $\Pi$. By [1, Corollary 2.3], $N N_{1}$ is abundant in every open subgroup that contains it, so $N N_{1}$ is abundant in $N N_{1} N_{2}$. Thus we can replace $\Pi$ by $N N_{1} N_{2}$ in order to assume that $\Pi=N N_{1} N_{2}$; it suffices to prove (ii) and (ii) for this $\Pi$. Lemma 3.6 gives open subgroups $E_{1}<E$ of $\Pi$ that contain $N N_{1}$ for which $f([\Pi: E]) \geq s \cdot\left[E: E_{1}\right]$ ! and $\left[E: E_{1}\right] \geq 3$. Then, $N_{1} \leq E$ and $E_{1} N_{2}=\Pi$.

The third case is the same as the second case, after exchanging the indices 1 and 2 .

In the last case we assume that $\left[\Pi:\left(N N_{1}\right) \cap\left(N N_{2}\right)\right]<\infty$ and $N$ is abundant in $\Pi$ I. In particular $N N_{i}$ is open in $\Pi$, so

$$
\left[N_{i}: N_{i} \cap N\right]=\left[N N_{i}: N\right]=\infty, \quad i=1,2 .
$$

Let $\Pi^{\prime}=\left(N N_{1}\right) \cap\left(N N_{2}\right)$. Then since $\Pi^{\prime}$ is open in $\Pi$, it follows that $N$ is abundant in $\Pi^{\prime}$. 
Put $N_{1}^{\prime}=N_{1} \cap \Pi^{\prime}$ and $N_{2}^{\prime}=N_{2} \cap \Pi^{\prime}$. Then $N N_{1}^{\prime}=N N_{2}^{\prime}=\Pi^{\prime}$. Since $\left[N_{i}: N_{i}^{\prime}\right]<\infty$, by (13), it follows that $\left[N_{i}^{\prime}: N_{i}^{\prime} \cap N\right]=\infty$.

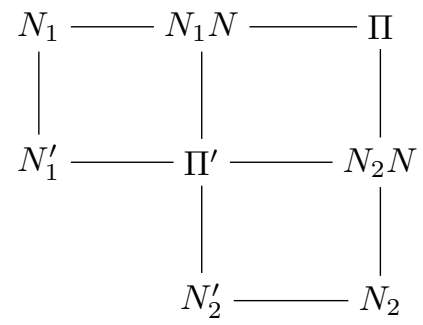

Replace $\Pi$ by $\Pi^{\prime}, N_{1}$ by $N_{1}^{\prime}$, and $N_{2}$ by $N_{2}^{\prime}$, if necessary, to assume that $N N_{1}=\Pi$ and $N N_{2}=\Pi$; it suffices to prove (i) and (ii) for this $\Pi$. Lemma 3.6 gives open subgroups $E_{1} \leq E$ of $\Pi$ containing $N$ with $f([\Pi: E]) \geq s \cdot\left[E: E_{1}\right]$ ! and $\left[E: E_{1}\right] \geq 3$. Meanwhile, for $i=1,2$,

$$
\Pi=N N_{i} \leq E_{1} N_{i} \leq \Pi,
$$

hence these subgroups satisfy (ii).

3.3. Proof of Theorem 1.2, Let $\Pi$ be a profinite surface group of genus $g \geq 2$. We start with two lemmas.

Lemma 3.7. A sparse subgroup of $\Pi$ is semi-free of countable rank.

Proof. Assume $N \leq \Pi$ is sparse. Since $\Pi$ is finitely generated, the rank of $N$ is at most $\aleph_{0}$. Thus it suffices to solve any finite split embedding problem $(\mu: N \rightarrow B, \alpha: A \rightarrow$ $B$ ) for $N$ [2, Lemma 3.4].

Choose an open normal subgroup $D \triangleleft \Pi$ with $D \cap N \leq \operatorname{ker} \mu$ and set $H=N D$. Then $H$ is open in $\Pi$ and $\mu$ extends to an epimorphism $\mu^{\prime}: H \rightarrow B$ by setting $\mu^{\prime}(n d)=\mu(n)$ for all $n \in N, d \in D$.

Since $N$ is sparse in $\Pi$, by [1, Lemma 2.2], there is an open subgroup $H_{0}$ of $H$ that contains $N$ such that $\left[\Pi: H_{0}\right] \geq 2|A|^{3}$ and every proper open subgroup $N \leq H_{1} \lesseqgtr H_{0}$ satisfies $\left[H_{0}: H_{1}\right]>|A|$. Note that $\mu_{0}=\left.\mu^{\prime}\right|_{H_{0}}$ is surjective, since $\mu^{\prime}\left(H_{0}\right) \geq \mu^{\prime}(N)=B$.

By Fact 2.1, we get that $H_{0}$ is a profinite surface group of genus

$$
g_{0}=\left[\Pi: H_{0}\right](g-1)+1>\left[\Pi: H_{0}\right] \geq 2|A|^{3} .
$$

By Lemma 2.2, the split embedding problem

$$
\left(\mu_{0}: H_{0} \rightarrow B, \alpha: A \rightarrow B\right)
$$

is properly solvable; let $\gamma: H_{0} \rightarrow A$ be a solution. It suffices to show that $\gamma(N)=A$, or equivalently $N \operatorname{ker} \gamma=H_{0}$, since then $\left.\gamma\right|_{N}$ is a solution of $(\mu, \alpha)$. Indeed, as $\left[H_{0}: N \operatorname{ker} \gamma\right] \leq\left[H_{0}: \operatorname{ker} \gamma\right] \leq|A|$, we have $N \operatorname{ker} \gamma=H_{0}$.

Lemma 3.8. Assume $N$ satisfies Condition ( $\diamond)$ in $\Pi$. Then $N$ is semi-free. 
Proof. Since $\Pi$ is finitely generated we get that $N$ is countably generated. Hence it suffices to show that every finite split embedding problem

$$
\mathcal{E}=\left(\mu_{1}: N \rightarrow G_{1}, \alpha_{1}: A \rtimes G_{1} \rightarrow G_{1}\right)
$$

with $A \neq 1$ is properly solvable.

Let $f(x)=\log x$ and take $N^{\prime}=\operatorname{ker} \mu_{1}$. Choose $r$ such that

$$
e^{r y} \geq 2|A|^{3 y} y^{3}, \forall y \geq 2 .
$$

By Condition $(\diamond)$ applied to $f, r$ and $N^{\prime}$, there exist normal subgroups $N_{1}, N_{2}$ of $\Pi$ such that $N_{1} \cap N_{2} \leq N,\left[N_{1}: N_{1} \cap N\right] \geq 3,\left[N_{2}: N_{2} \cap N\right] \geq 2$ and a diagram of subgroups

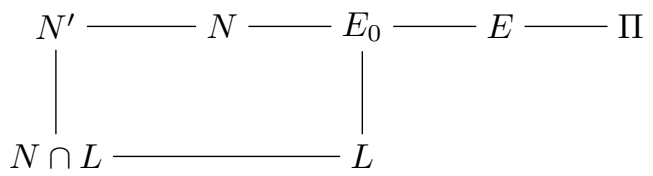

satisfying the conditions (9). In particular, $9 \mathrm{e}$ is equivalent to $[\Pi: E] \geq e^{r \cdot[E: L]}$. Then $E$ is a surface group of genus

$$
g_{0}=[\Pi: E](g-1)+1 \geq e^{r \cdot[E: L]} \geq 2|A|^{3[E: L]}[E: L]^{3} .
$$

Let $N_{i}^{\prime}=N_{i} \cap E$. We apply Lemma 3.2 with $E$ replacing $\Pi$ and $N_{i}^{\prime}$ replacing $N_{i}$. Let $G=E / L, G_{0}=N L / L \cong N / N \cap L$, and

$$
\mathcal{E}_{\text {ind }}=\left(\mu: E \rightarrow G, \alpha: A \text { ใ }_{G_{0}} G \rightarrow G\right)
$$

the induced embedding problem. We claim that the conditions (ii)-(iv) of the lemma are satisfied. Indeed, $L \cap N \leq N^{\prime}$ by the diagram, so we have (i). Since $N L \leq E_{0} \leq E$ we have

$$
\left[N_{i}^{\prime} N L: N L\right]=\left[N_{i}^{\prime}: N_{i}^{\prime} \cap N L\right]=\left[N_{i} \cap E:\left(N_{i} \cap E\right) \cap N L\right] \geq\left[N_{i} \cap E: N_{i} \cap E_{0}\right] .
$$

So (9c) and (9d) imply (ii) and (iii). Finally, by (9c) we have

$$
[E: N L] \geq\left[E: E_{0}\right] \geq\left[N_{1} \cap E: N_{1} \cap E_{0}\right] \geq 3 .
$$

So (iv) is satisfied.

Now by (15) we have that $g_{0} \geq 2\left|A \imath_{G_{0}} G\right|^{3}$, hence by Lemma $2.2, \mathcal{E}_{\text {ind }}$ is properly solvable, and thus by Lemma 3.2 so is $\mathcal{E}$.

Proof of Theorem 1.2. As explained at the beginning of Section 3.2, we can assume that $\left[N_{1} N: N\right]=\infty$.

Note that by Lemma 3.8 if one of the conditions of Proposition 3.4 is satisfied, then $N$ is semi-free. Hence we assume that none of them holds.

If $\left[\Pi:\left(N N_{1}\right) \cap\left(N N_{2}\right)\right]<\infty$, then the negation of Condition 10d of Proposition 3.4 gives that $N$ is sparse in $\Pi$. Hence $N$ is semi-free of countable rank (Lemma 3.7). Thus we may assume that $\left[\Pi:\left(N N_{1}\right) \cap\left(N N_{2}\right)\right]=\infty$. W.l.o.g. $\left[\Pi: N N_{1}\right]=\infty$. Then the negation of $(10 \mathrm{a}) \vee(10 \mathrm{~b})$ ) gives that $N N_{1}$ is sparse in $\Pi$. Then Lemma 3.7 gives that $N N_{1}$ is semi-free of countable rank.

Put $N_{2}^{\prime}=\left(N N_{1}\right) \cap N_{2}$. Then $N_{1}, N_{2}^{\prime} \triangleleft N N_{1}, N_{1} \cap N_{2}^{\prime} \leq N$ and $N_{1} \not \leq N$. If $N_{2}^{\prime} \not \leq N$, then the diamond theorem for semi-free groups (2, Main Theorem VI]) gives that $N$ is semi-free of countable rank. 
We are left with the case $N_{2}^{\prime} \leq N$. Then $N=N N_{1} \cap N N_{2}$. By the negation of

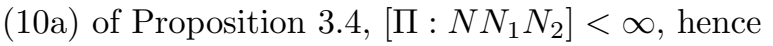

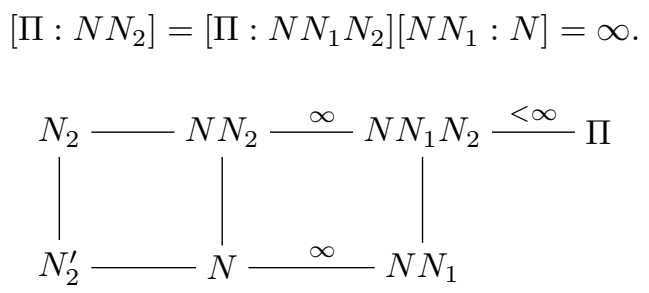

The negation of 10c) of Proposition 3.4 gives that $N N_{2}$ is sparse in $\Pi$ and hence in the open subgroup $N N_{1} N_{2}$ of $\Pi$ ([1, Corollary 2.3]). But since $N N_{1} / N_{2}^{\prime} \cong N N_{1} N_{2} / N_{2}$, this implies that $N$ is sparse in the semi-free group $N N_{1}$, and hence $N$ is semi-free of countable rank ([1, Lemma 2.4]).

3.4. Proof of Theorem 1.1. Since $[\Pi: N]=\prod_{p} p^{\infty}, N$ is projective [8, Proposition 1.2]. By Theorem 1.2, $N$ is semi-free. Therefore, by [2, Theorem 3.6] $N$ is free.

\section{Applications}

4.1. Proof of Theorem 1.4. Let $\Pi$ be a surface group of genus $g \geq 2$ and let $N \triangleleft \Pi$ be a normal subgroup of infinite index such that $\Pi / N$ is not hereditary just infinite . We need to prove that $N$ is contained in a semi-free normal subgroup.

If there exists a normal subgroup $N \supsetneqq M \triangleleft \Pi$ with $[\Pi: M]=\infty$, then there exists $N \leq U \triangleleft \Pi$ open in $\Pi$ such that $M \cap U \neq M$ (recall that $N$ is the intersection of all open subgroups containing it). So $M \cap U$ is semi-free by Theorem 1.2 , and we are done.

Therefore we can assume that $J=\Pi / N$ is just infinite. By [4, Theorem 3(b)], there exists an open normal subgroup $J_{0}$ of $J$ such that either $J_{0}$ is hereditarily just infinite or $J_{0}=A \times B$, where $A$ and $B$ are infinite groups. The former is not possible, since if $U$ is an open normal subgroup of $J$ with a proper infinite quotient, then $U \cap J_{0}$ is an open normal subgroup of $J_{0}$ with a proper infinite quotient.

Let $\Pi_{0}, K_{1}, K_{2}$ be the respective preimages of $J_{0}, A, B$ under the map $\Pi \rightarrow J$. Then $\Pi_{0}$ is a surface group of genus $\geq 2$ and $N=K_{1} \cap K_{2}$. So by Theorem $1.2, N$ is semi-free.

Remark 4.1. Let $N$ be a normal subgroup of $\Pi$ such that $\Pi / N$ is hereditarily just infinite. We do not know whether $N$ is necessarily semi-free.

4.2. Proof of Corollary 1.3. Let $\Pi$ be a surface group of genus at least $2, N$ a normal subgroup of $\Pi$ of infinite index, and $M$ a proper open subgroup of $N$. There exists an open normal subgroup $U \triangleleft \Pi$ such that $U \cap N \leq M$. Note that $U$ is not contained in $M$ because $[\Pi: M]=\infty$. By Theorem $1.2, M$ is semi-free. 
4.3. Some examples. Let $\Pi$ be a surface group of genus at least 2 and $N$ a closed subgroup of infinite index. The following result provides many interesting examples of semi-free subgroups of a surface group.

Proposition 4.2. If $N \triangleleft \Pi$ and if every open subgroup of $\Pi / N$ can be generated by $d$ elements, for some $d \geq 1$, or if $N$ is sparse in $\Pi$, then $N$ is semi-free.

Proof. Let

$$
\mathcal{E}(N)=\left(\mu_{0}: N \rightarrow A, \alpha_{0}: C \rtimes A \rightarrow A\right)
$$

be a FSEP for $N$. Since $N$ is countably generated, it suffices to show that $\mathcal{E}(N)$ has a proper solution. Assume first that every open subgroup of $\Pi / N$ is generated by $d$ elements. Choose $r, n \geq 1$ such that $n>((|C||A|) !)^{d}$ and $r \geq 2|A|^{3}|C|^{3 n}$.

Choose $L \triangleleft \Pi$ to be an open subgroup of $\Pi$ such that $L \cap N \leq \operatorname{ker} \mu_{0}$. Choose $\tilde{\Pi}$ be an open subgroup of $\Pi$ such that $N \leq \tilde{\Pi} \leq L N$ and such that $[\Pi: \tilde{\Pi}] \geq r$. Then we can extend $\mu_{0}$ to $\mu$ : $\tilde{\Pi} \rightarrow A$ by $\mu(n l)=\mu_{0}(n)$, for every $n \in N, l \in L$, for which $n l \in \tilde{\Pi}$. By Fact 2.1 the genus of $\tilde{\Pi}$ is at least $r$. Without loss of generality we can replace $\Pi$ with $\tilde{\Pi}$ to assume $\mu_{0}$ can be extended to $\mu: \Pi \rightarrow A$ and $g \geq r$. (Note that the rank of $\tilde{\Pi} / N$ is bounded by the rank of $\Pi / N$.)

Consider the FSEP

$$
\mathcal{E}_{n}(\Pi)=\left(\mu: \Pi \rightarrow A, \alpha: C^{n} \rtimes A \rightarrow A\right),
$$

where $A$ acts component-wise on $C^{n}$. Since $g \geq r \geq 2|A|^{3}|C|^{3 n}$, by Lemma 2.2 , there exists a proper solution $\Psi: \Pi \rightarrow C^{n} \rtimes A$ of $\mathcal{E}_{n}(\Pi)$. For each $i=1, \ldots, n$, let $\psi_{i}$ be the composition of $\Psi$ with the projection $C^{n} \rtimes A \rightarrow C \rtimes A$ on the $i$ th coordinate. Let $L_{i}=\operatorname{ker} \psi_{i}$. Then $L_{i} L_{j}=\operatorname{ker} \mu$, for every $i \neq j$.

If $L_{i} N=\Pi$ for some $i$, then $\psi_{i}(N)=C \rtimes A$, so $\left.\psi_{i}\right|_{N}$ is a proper solution of $\mathcal{E}(N)$, and we are done.

Otherwise, assume that $L_{i} N \neq \Pi$ for every $i$. But since $\left(L_{i} N\right)\left(L_{j} N\right)=\left(L_{i} L_{j}\right) N=$ ker $\mu N=\Pi$, we get that $L_{i} N$ are distinct subgroups of index $\leq|C||A|$. So $\Pi / N$ has at least $n>((|C||A|) !)^{d}$ open subgroups of index $\leq|C||A|$. This is a contradiction because each such a subgroup induces a distinct homomorphism to the symmetric group $S_{|C||A|}$ defined by the action on the cosets, and the number of these homomorphisms is bounded by $((|C||A|) !)^{d}$.

Next assume that $N$ is sparse in $\Pi$. Replace $\Pi$ by an open subgroup $\tilde{\Pi}$ of index $[\Pi: \tilde{\Pi}] \geq 2|C|^{3}|A|^{3}$ that contains $N$ such that $\tilde{\Pi}$ has no proper subgroups of index $\leq|C||A|$ that contain $N$. Then arguing as above with $n=1$, we get that $L_{1} N \leq \Pi$ and $\left[\Pi: L_{1} N\right] \leq|C||A|$, so $L_{1} N=\Pi$. So $\left.\psi_{1}\right|_{N}$ is a proper solution of $\mathcal{E}(N)$.

Examples 4.3. Each of the following conditions implies that $N$ is semi-free.

(1) $\Pi / N=\mathbb{Z}_{p}$ (every subgroup is cyclic)

(2) $\Pi / N=K_{1} \times K_{2}\left(N\right.$ is the intersection of the preimages of $K_{1}, K_{2}$ in $\Pi$, hence by Theorem 1.2 is semi-free).

(3) $\Pi / N$ is abelian $\left(\Pi / N\right.$ is either $\mathbb{Z}_{p}$ or direct product).

(4) $\Pi / N$ is pro-nilpotent but not pro- $p(\Pi / N$ is a direct product $)$.

(5) $[\Pi: N]=\prod_{p} p^{n(p)}$, where $0 \leq n(p)<\infty$ (this implies that $N$ is sparse in $\Pi$ ).

Notice that (2) gives a new proof that the congruence kernel of an arithmetic lattice in $S L_{2}(\mathbb{R})$ is a free profinite group of countable rank, see [8] for more details. 


\section{Acknowledgements}

We thank the referee for the many helpful remarks that greatly improved the paper. The first author is an Alexander von Humboldt fellow. The last author is partially supported by CNPq.

\section{References}

1. Lior Bary-Soroker, Diamond theorem for a finitely generated free profinite group, Math. Ann. 336 (2006), no. 4, 949-961

2. Lior Bary-Soroker, Dan Haran, and David Harbater, Permanence criteria for semi-free profinite groups, vol. 348, no. 3, 539-563, doi:10.1007/s00208-010-0484-8

3. $\quad$ Field arithmetic, third ed., Ergebnisse der Mathematik und ihrer Grenzgebiete. 3. Folge. A Series of Modern Surveys in Mathematics [Results in Mathematics and Related Areas. 3rd Series. A Series of Modern Surveys in Mathematics], vol. 11, Springer-Verlag, Berlin, 2008, Revised by Jarden

4. R. I. Grigorchuk, Just infinite branch groups, New horizons in pro-p groups, Progr. Math., vol. 184, Birkhäuser Boston, Boston, MA, 2000, pp. 121-179

5. David Harbater and Katherine F. Stevenson, Local Galois theory in dimension two, Adv. Math. 198 (2005), no. 2, 623-653

6. Amílcar Pacheco, Katherine F. Stevenson, and Pavel Zalesskii, Normal subgroups of the algebraic fundamental group of affine curves in positive characteristic, Math. Ann. 343 (2009), no. 2, 463-486

7. Luis Ribes and Pavel Zalesskii, Profinite groups, second ed., Ergebnisse der Mathematik und ihrer Grenzgebiete. 3. Folge. A Series of Modern Surveys in Mathematics [Results in Mathematics and Related Areas. 3rd Series. A Series of Modern Surveys in Mathematics], vol. 40, Springer-Verlag, Berlin, 2010

8. P. A. Zalesskii, Profinite surface groups and the congruence kernel of arithmetic lattices in $\mathrm{SL}_{2}(\mathbf{R})$, Israel J. Math. 146 (2005), 111-123

Institut für Experimentelle Mathematik, Universität Duisburg-Essen, Ellernstrasse 29, D-45326 Essen, Germany

E-mail address: lior.bary-soroker@uni-due.de

Department of Mathematics, California State University Northridge, 18111 Nordhoff St Northridge, CA 91330-8313, USA

E-mail address: katherine.stevenson@csun.edu

Department of Mathematics, University of Brasília, Brasília-DF 70910-900, Brazil

E-mail address: pz@mat.unb.br 